\title{
STATIC AND KINEMATIC SHAKEDOWN THEOREMS IN DIFFUSION-INDUCED PLASTICITY ${ }^{1}$
}

\author{
Michael Peigney \\ Laboratoire Navier, Ecole des Ponts ParisTech, Université Gustave Eiffel, CNRS, Marne la Vallee, France \\ e-mail: michael.peigney@polytechnique.org
}

\begin{abstract}
We present shakedown theorems applying to cyclically loaded media in which plastic flow and diffusion of guest atoms are coupled. The presented theorems are in the spirit of Melan and Koiter theorems in plasticity. They allow one to estimate the loading parameters for which elastic shakedown occurs, i.e. for which the plastic strain stabilizes to a time-independent limit. An application related to lithium-ion batteries is presented.
\end{abstract}

Keywords: plasticity, shakedown, diffusion-induced stress, lithium-ion batteries

\section{Introduction}

In this paper, cyclically loaded media are studied in the framework of diffusion-induced plasticity. In the media considered, plasticity and diffusion act as two concurrent nonlinear and dissipative processes. Elastic shakedown corresponds to the situation in which plastic dissipation is bounded. Accordingly, the medium is expected to reach a steady state cycle in which diffusion is the only active source of dissipation. Electrode materials in lithium-ion batteries are an example of the medium in which stress and plastic flow are generated by diffusion of guest atoms. For such applications, elastic shakedown is favorable for the electromechanical performance because the hysteresis in the charge-voltage cycle is reduced (Brassart et al., 2013).

In standard plasticity (without diffusion), the Melan theorem gives a sufficient condition on the loading for ensuring that elastic shakedown occurs, independently of any residual stress that may exist in the initial state (Melan, 1936; Koiter, 1960). The Melan theorem (also known as the static shakedown theorem) has been extended to several more complex behaviors than perfect plasticity, see e.g. Peigney (2010, 2014a,b), Pham (2017), Klarbring et al. (2017) for relatively recent examples. In particular, the Melan theorem has been extended to continuous media in which plasticity and diffusion are coupled (Peigney, 2018). In practice, that theorem allows one to obtain lower bounds on the set of loading parameters for which elastic shakedown occurs.

In this paper, we use convex duality to generate upper bounds, resulting in a kinematic shakedown theorem formally similar to Koiter's theorem in standard plasticity (without diffusion). An application related to electrode particles in lithium-ion batteries is presented.

\section{Diffusion-induced plasticity in cyclically loaded media}

\subsection{Conservation equations}

Consider a deformable continuum occupying a domain $\Omega$ in which guest atoms diffuse. A normal flux $J$ of guest atoms is prescribed on a part $\Gamma_{J}$ of the boundary. On $\Gamma_{\mu}=\partial \Omega-\Gamma_{J}$,

\footnotetext{
${ }^{1}$ This work is related to a paper presented at PCM-CMM-2019
} 
the chemical potential $\mu$ is prescribed to take a given value $M$. Tractions $\mathbf{T}$ are prescribed on a part $\Gamma_{T}$ of the boundary and displacements $\mathbf{U}$ are prescribed on $\Gamma_{u}=\partial \Omega-\Gamma_{T}$.

Mass conservation leads to the diffusion equation

$$
\dot{c}+\operatorname{div} \jmath=0 \text { in } \Omega
$$

where $c$ is the concentration of guest atoms and $\jmath$ is the flux. Equation (2.1) is complemented by the boundary conditions

$$
\mu=M \text { on } \Gamma_{\mu} \quad \jmath \cdot \mathbf{n}=J \text { on } \Gamma_{J}
$$

Assuming deformation to be much faster than diffusion, the stress field $\boldsymbol{\sigma}$ satisfies the equilibrium equation

$$
\operatorname{div} \boldsymbol{\sigma}+\mathbf{f}=0 \text { in } \Omega
$$

where $\mathbf{f}$ is the body force. Equation (2.3) is complemented by the boundary conditions

$$
\boldsymbol{\sigma} \cdot \mathbf{n}=\mathbf{T} \text { on } \Gamma_{T} \quad \mathbf{u}=\mathbf{U} \text { on } \Gamma_{u}
$$

In the equations above, $M, J, \mathbf{f}, \mathbf{U}, \mathbf{T}$ are functions of the location $\mathbf{x}$ and time $t$ that define the applied chemo-mechanical loading history. They are assumed to be periodic in time, with the same period $T$. Functions that are periodic in time with a period $T$ are referred to as $T$-periodic in the following.

\subsection{Constitutive equations}

An elastic-perfectly plastic constitutive material is considered. Following the thermodynamic framework of Larché and Cahn (1973), the local state of the material is described by the total linearized strain $\varepsilon$, the (deviatoric) plastic strain $\varepsilon^{p}$ and the concentration $c$ of guest atoms. The free energy $w$ of the material is taken as a quadratic function of the form

$$
w\left(\varepsilon, \varepsilon^{p}, c\right)=\frac{1}{2}\left(\varepsilon-\varepsilon^{p}\right): \mathbb{L}:\left(\varepsilon-\varepsilon^{p}\right)+\frac{1}{2} k c^{2}+c \mathbf{A}:\left(\varepsilon-\varepsilon^{p}\right)+c \mu_{0}
$$

where $\mathbb{L}$ is the elasticity tensor and $\mathbf{A}$ is a symmetric second-order tensor accounting for the coupling between the elastic strain $\varepsilon-\varepsilon^{p}$ and the concentration $c$. The material parameter $k$ is assumed to satisfy the relation $k>\mathbf{A}: \mathbb{L}^{-1}: \mathbf{A}$ which ensures that the function $w$ in $(2.5)$ is convex. From (2.5), we obtain the constitutive relations

$$
\boldsymbol{\sigma}=\frac{\partial w}{\partial \boldsymbol{\varepsilon}}=\mathbb{L}:\left(\varepsilon-\varepsilon^{p}\right)+c \mathbf{A} \quad \mu=\frac{\partial w}{\partial c}=\mu_{0}+k c+\mathbf{A}:\left(\varepsilon-\varepsilon^{p}\right)
$$

Note that the concentration $c$ has a linear influence on the stress, in a way similar to the thermal stress. Similarly, the chemical potential $\mu$ depends linearly on the elastic strain.

It is insightful to look at the thermodynamical dissipation in the type of the medium considered. Considering for instance the case where $J=0$ and $\Gamma_{J}=\partial \Omega$ (so that the continuum in $\Omega$ forms a closed system), the second law of thermodynamics states that

$$
\dot{W}-P_{\text {ext }} \geqslant 0
$$

where $\dot{W}=\int_{\Omega} \dot{w} d v$ and $P_{\text {ext }}$ is the external power. Using (2.6) together with equilibrium equation (2.3) and diffusion equation (2.1), the dissipation $\dot{W}-P_{\text {ext }}$ can be rewritten as

$$
\dot{W}-P_{e x t}=\int_{\Omega} \boldsymbol{\sigma}: \dot{\boldsymbol{\varepsilon}}^{p}-\nabla \mu \cdot \jmath d v
$$


Therefore, there are two sources of dissipation, namely the plastic dissipation $\int_{\Omega} \boldsymbol{\sigma}: \dot{\boldsymbol{\varepsilon}}^{p}$ and the diffusion-related dissipation $\int_{\Omega}-\nabla \mu \cdot \jmath d v$. The law of diffusion and the plasticity flow rule complement constitutive equations (2.6) in such fashion that the second law of thermodynamics (2.7) is respected. In more detail, the flux $\int$ of guest atoms is assumed to obey the relation

$$
\jmath=-\partial \psi(\nabla \mu)
$$

where $\psi$ is a positive, differentiable and strictly convex function such that $\psi(0)=0$. Under such assumptions, we have $-\nabla \mu \cdot \jmath \geqslant \psi(\nabla \mu)-\psi(0) \geqslant 0$.

A normality flow rule is assumed for the plastic behavior, i.e.

$$
\dot{\boldsymbol{\varepsilon}}^{p}=\lambda \frac{\partial f}{\partial \boldsymbol{\sigma}}(\boldsymbol{\sigma}) \quad \text { with } \quad \lambda \geqslant 0, \quad f(\boldsymbol{\sigma}) \leqslant 0, \quad \lambda f(\boldsymbol{\sigma})=0
$$

where $f$ is a differentiable, strictly convex function of the deviatoric stress such that $f(0)<0$. Such assumptions guarantee that $\boldsymbol{\sigma}: \dot{\boldsymbol{\varepsilon}}^{p} \geqslant 0$. The elasticity domain of the material is denoted by $\mathcal{C}$, i.e.

$$
\mathcal{C}=\{\boldsymbol{\sigma}: f(\boldsymbol{\sigma}) \leqslant 0\}
$$

\subsection{Evolution equation for $(\sigma, \mu)$}

We denote by $\mathcal{K}(\mathbf{f}, \mathbf{T}, M)$ the set of stress and chemical potential fields that are compatible with data $(\mathbf{f}, \mathbf{T}, M)$, i.e.

$$
\mathcal{K}(\mathbf{f}, \mathbf{T}, M)=\left\{(\boldsymbol{\sigma}, \mu): \operatorname{div} \boldsymbol{\sigma}+\mathbf{f}=\mathbf{0} \text { in } \Omega, \boldsymbol{\sigma} \cdot \mathbf{n}=\mathbf{T} \text { on } \Gamma_{T}, \mu=M \text { on } \Gamma_{\mu}\right\}
$$

The set $\mathcal{K}(0,0,0)$ is more simply denoted by $\mathcal{K}_{0}$. Let $\left(\boldsymbol{\sigma}, \mu, \mathbf{u}, c, \jmath, \boldsymbol{\varepsilon}^{p}\right)$ be a solution to the evolution problem defined by the equations formulated in Sections 2.1 and 2.2. It can be shown that

$$
\begin{aligned}
& (\boldsymbol{\sigma}, \mu) \in \mathcal{K}(\mathbf{f}, \mathbf{T}, M) \\
& \langle(\dot{\boldsymbol{\sigma}}, \dot{\mu}),(\boldsymbol{\rho}, \nu)\rangle=-\int_{\Omega}\left(\dot{\boldsymbol{\varepsilon}}^{p}: \boldsymbol{\rho}+\partial \psi(\nabla \mu) \cdot \nabla \nu\right) d \omega+\int_{\Gamma_{u}} \dot{\mathbf{U}} \cdot \boldsymbol{\rho} \cdot \mathbf{n} d S \\
& -\int_{\Gamma_{J}} J \nu d S \quad \forall(\boldsymbol{\rho}, \nu) \in \mathcal{K}_{0}
\end{aligned}
$$

Setting $\mathbf{B}=\mathbf{A}: \mathbb{L}^{-1}$ and $k^{\prime}=k-\mathbf{A}: \mathbb{L}^{-1}: \mathbf{A}$, the scalar product $\langle\cdot, \cdot\rangle$ in $(2.11)$ is defined by

$$
\left\langle(\boldsymbol{\sigma}, \mu),\left(\boldsymbol{\sigma}^{\prime}, \mu^{\prime}\right)\right\rangle=\int_{\Omega} \boldsymbol{\sigma}: \mathbb{L}^{-1}: \boldsymbol{\sigma}^{\prime}+\frac{1}{k^{\prime}}\left((\mathbf{B}: \boldsymbol{\sigma})\left(\mathbf{B}: \boldsymbol{\sigma}^{\prime}\right)+\mu \mu^{\prime}-\mu^{\prime} \mathbf{B}: \boldsymbol{\sigma}-\mu \mathbf{B}: \boldsymbol{\sigma}^{\prime}\right) d \omega
$$

Since $\dot{\boldsymbol{\varepsilon}}^{p}$ is directly related to the stress via (2.10), Eq. (2.11) can be interpreted as an evolution equation for the stress and chemical potential fields $(\sigma, \mu)$. Some properties of evolution equation (2.11) have been studied in Peigney (2018). For our purpose, one of the main result is that any solution to (2.11) converges towards a cyclic steady state in which $\dot{\varepsilon}^{p}, \boldsymbol{\sigma}, \mu, \jmath$ are $T$-periodic. Moreover, the values of $\dot{\boldsymbol{\varepsilon}}^{p}, \dot{\boldsymbol{\sigma}}, \dot{\mu}, \jmath$ on the cyclic steady state are unique, i.e. independent of the initial conditions. Elastic shakedown corresponds to the special case where $\dot{\boldsymbol{\varepsilon}}^{p}=0$ in the cyclic steady state. This implies that the total dissipation on the cyclic steady state reduces to the diffusion-related dissipation, see Eq. (2.8). 


\subsection{Case of elasto-diffusion}

Let us consider the purely elasto-diffusive problem obtained by discarding plastic flow in the original problem. That fictitious problem is defined by the very same equations as before, except that the variable $\varepsilon^{p}$ is removed. As a particular case of (2.11), the stress and chemical potential fields $(\tilde{\boldsymbol{\sigma}}, \tilde{\mu})$ of the purely elasto-diffusive problem satisfy

$$
\begin{aligned}
& (\tilde{\boldsymbol{\sigma}}, \widetilde{\mu}) \in \mathcal{K}(\mathbf{f}, \mathbf{T}, M) \\
& \langle(\dot{\tilde{\boldsymbol{\sigma}}}, \dot{\tilde{\mu}}),(\boldsymbol{\rho}, \nu)\rangle=-\int_{\Omega} \partial \psi(\nabla \widetilde{\mu}) \cdot \nabla \nu d \omega+\int_{\Gamma_{u}} \dot{\mathbf{U}} \cdot \boldsymbol{\rho} \cdot \mathbf{n} d S-\int_{\Gamma_{J}} J \nu d S \quad \forall(\boldsymbol{\rho}, \nu) \in \mathcal{K}_{0}
\end{aligned}
$$

T-periodic solutions to (2.13) play an important role in the shakedown analysis as detailed later on. Although there is not uniqueness, $T$-periodic solutions to (2.13) are quite similar to one another as they differ by constant fields. More precisely, if $\left(\boldsymbol{\sigma}^{E}, \mu^{E}\right)$ denotes a particular $T$-periodic solution to (2.13), then the whole family of $T$-periodic solutions to elasto-diffusive equation (2.13) is

$$
\left\{\left(\boldsymbol{\sigma}^{E}+\widetilde{\boldsymbol{\rho}}, \mu^{E}+\widetilde{\eta}\right):(\widetilde{\boldsymbol{\rho}}, \widetilde{\eta}) \in \mathcal{K}_{0} ; \widetilde{\boldsymbol{\rho}} \text { time-independent; } \widetilde{\eta} \text { constant }\right\}
$$

\section{Static shakedown theorem in coupled plasticity-diffusion}

Let $\mathcal{A}_{0}$ be the set of self-equilibrated stress fields, i.e.

$$
\mathcal{A}_{0}=\left\{\boldsymbol{\rho}: \operatorname{div} \boldsymbol{\rho}=0 \text { in } \Omega, \boldsymbol{\rho} \cdot \mathbf{n}=0 \text { on } \Gamma_{T}\right\}
$$

Both a necessary and a sufficient condition for shakedown have been obtained in Peigney (2018). They read as follows:

Shakedown condition 1 (necessary) If shakedown occurs, then there exists a T-periodic solution $(\tilde{\boldsymbol{\sigma}}, \widetilde{\mu})$ to purely elasto-diffusive equation (2.13) that satisfies $f(\tilde{\boldsymbol{\sigma}}) \leqslant 0$.

Shakedown condition 2 (sufficient) If there exists a time-independent stress field $\boldsymbol{\rho} \in \mathcal{A}_{0}$ such that $f\left(\boldsymbol{\sigma}^{E}(\mathbf{x}, t)+\boldsymbol{\rho}(\mathbf{x})\right)<0$ for all $(\mathbf{x}, t) \in \Omega \times[0, T]$, then shakedown occurs whatever the initial state.

Although the statement of shakedown condition 2 above is similar to the Melan theorem, we emphasize that its proof largely differs from the standard proof used in Melan theorem for pure plasticity.

Shakedown condition 2 motivates the introduction of a 'static security coefficient' $m_{S}$ as

$$
\begin{aligned}
& m_{S}=\sup \left\{m: \text { there exists } \boldsymbol{\rho} \in \mathcal{A}_{0} \text { such that } f\left(\boldsymbol{\rho}(\mathbf{x})+m \boldsymbol{\sigma}^{E}(\mathbf{x}, t)\right) \leqslant 0\right. \\
& \text { for all }(\mathbf{x}, t) \in \Omega \times[0, T]\}
\end{aligned}
$$

The value of $m_{S}$ indeed determines the shakedown behavior according to the following rules

$$
\left\{\begin{array}{l}
m_{S}>1 \Longrightarrow \text { shakedown occurs } \\
m_{S}<1 \Longrightarrow \text { shakedown does not occur }
\end{array}\right.
$$

Let us briefly justify (3.2). Firstly, consider the case $m_{S}>1$ and let $m>1, \rho \in \mathcal{A}_{0}$ be such $f\left(\boldsymbol{\rho}(\mathbf{x})+m \boldsymbol{\sigma}^{E}(\mathbf{x}, t)\right) \leqslant 0$ for all $(\mathbf{x}, t) \in \Omega \times[0, T]$. Since $f(0)=0$ and $f$ is strictly convex, we have

$$
f\left(\frac{\boldsymbol{\rho}}{m}+\boldsymbol{\sigma}^{E}\right)<\frac{1}{m} f\left(\boldsymbol{\rho}+m \boldsymbol{\sigma}^{E}\right) \leqslant 0
$$


Since $\boldsymbol{\rho} / m \in \mathcal{A}_{0}$, shakedown condition 2 applies and ensures that shakedown occurs. If now $m_{S}<1$, no time-independent stress field $\boldsymbol{\rho} \in \mathcal{A}_{0}$ can be found in such fashion that $f(\boldsymbol{\rho}(\mathbf{x})+$ $\left.\boldsymbol{\sigma}^{E}(\mathbf{x}, t)\right) \leqslant 0$ for all $(\mathbf{x}, t) \in \Omega \times[0, T]$. Therefore, by $(2.14)$ there is no $T$-periodic solution $(\widetilde{\boldsymbol{\sigma}}, \widetilde{\mu})$ to the pure elasto-diffusive problem such that $f(\widetilde{\boldsymbol{\sigma}}) \leqslant 0$ on $\Omega \times[0, T]$, which is a prerequisite for shakedown to occur (see shakedown condition 1 above).

In practice, lower bounds $m_{S}^{-}$on $m_{S}$ are obtained by choosing particular self-equilibrated stress fields in (3.1). If such a lower bound $m_{S}^{-}$verifies $1 \leqslant m_{S}^{-}$, then shakedown occurs as a consequence of (3.2).

\section{Kinematic shakedown theorem}

Our objective is now to obtain upper bounds on $m_{S}$. In perfect plasticity (without diffusion), Koiter's theorem allows such upper bounds to be obtained (Koiter, 1960). We recall that the reasoning used in Koiter's theorem consists in bounding the total dissipation. In diffusion-induced plasticity, bounding the total dissipation amounts to bounding the plastic dissipation and the diffusion-related dissipation, meaning that the medium reaches an elastic cyclic steady state in which the concentration is time-independent. Here we are interested in situations in which the plastic strain - but not the concentration - stabilizes to a limit, so that diffusion is the only active source of dissipation in the cyclic steady state. To obtain relevant bounds in such a case, we use a different line of arguments than those used by Koiter (1960). Specifically, the strategy that we employ consists in using convex duality, as used for instance by Nguyen (2003).

Let $\mathcal{A}$ be the set of triplets $\left(m, \boldsymbol{\rho}^{*}, \widetilde{\boldsymbol{\sigma}}\right)$ such that $\boldsymbol{\rho}^{*}(\mathbf{x})$ is a time-independent self-equilibrated stress field and $\tilde{\boldsymbol{\sigma}}(\mathbf{x}, t) \in \mathcal{C}$ for all $(\mathbf{x}, t)$. From (3.1) we have

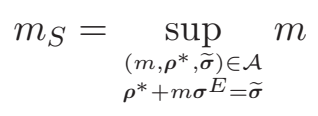

This is a constrained maximization problem over the convex set $\mathcal{A}$. Denote by $\mathcal{L}$ the corresponding lagrangian, i.e.

$$
\mathcal{L}\left(m, \boldsymbol{\rho}^{*}, \tilde{\boldsymbol{\sigma}} ; \mathbf{d}\right)=m+\int_{\Omega} \int_{0}^{T} \mathbf{d}(\mathbf{x}, t):\left(\widetilde{\boldsymbol{\sigma}}(\mathbf{x}, t)-\boldsymbol{\rho}^{*}(\mathbf{x})-m \boldsymbol{\sigma}^{E}(\mathbf{x}, t)\right) d v d t
$$

where the second-order tensor $\mathbf{d}$ plays the role of a Lagrange multiplier associated to the constraint $\boldsymbol{\rho}^{*}+m \boldsymbol{\sigma}^{E}=\widetilde{\boldsymbol{\sigma}}$. We have

$$
m_{S}=\sup _{\left(m, \boldsymbol{\rho}^{*}, \widetilde{\boldsymbol{\sigma}}\right) \in \mathcal{A}} \inf _{\mathbf{d}} \mathcal{L}\left(m, \boldsymbol{\rho}^{*}, \widetilde{\boldsymbol{\sigma}} ; \mathbf{d}\right)
$$

The min-max inequality gives

$$
\sup _{\left(m, \boldsymbol{\rho}^{*}, \widetilde{\sigma}\right) \in \mathcal{A}} \inf _{\mathbf{d}} \mathcal{L} \leqslant \inf _{\mathbf{d}} \sup _{\left(m, \boldsymbol{\rho}^{*}, \widetilde{\boldsymbol{\sigma}}\right) \in \mathcal{A}} \mathcal{L}
$$

Equation (4.2) implies that

$$
m_{S} \leqslant m_{K}
$$

where

$$
m_{K}=\inf _{\mathbf{d}} \sup _{\left(m, \boldsymbol{\rho}^{*}, \widetilde{\boldsymbol{\sigma}}\right) \in \mathcal{A}} \mathcal{L}\left(m, \boldsymbol{\rho}^{*}, \widetilde{\boldsymbol{\sigma}} ; \mathbf{d}\right)
$$


Let us rewrite the coefficient $m_{K}$ in a more explicit form. For a given $\mathbf{d}$, the definition (4.1) yields

$$
\begin{aligned}
& \sup _{\left(m, \boldsymbol{\rho}^{*}, \widetilde{\boldsymbol{\sigma}}\right) \in \mathcal{A}} \mathcal{L}=\sup _{m} m\left(1-\int_{\Omega} \int_{0}^{T} \mathbf{d}(\mathbf{x}, t): \boldsymbol{\sigma}^{E}(\mathbf{x}, t) d v d t\right) \\
& \quad+\sup _{\widetilde{\boldsymbol{\sigma}} \in \mathcal{C}} \int_{\Omega} \int_{0}^{T} \mathbf{d}(\mathbf{x}, t): \widetilde{\boldsymbol{\sigma}}(\mathbf{x}, t) d v d t+\sup _{\boldsymbol{\rho}^{*} \in \mathcal{A}_{0}}-\int_{\Omega} \mathbf{E}^{p}(\mathbf{x}): \boldsymbol{\rho}^{*}(\mathbf{x}) d v d t
\end{aligned}
$$

where $\mathbf{E}^{p}=\int_{0}^{T} \mathbf{d}(\mathbf{x}, t) d t$. Let us examine each term in the right-hand side of (4.4). We clearly have

$$
\sup _{m} m\left(1-\int_{\Omega} \int_{0}^{T} \mathbf{d}: \boldsymbol{\sigma}^{E} d v d t\right)= \begin{cases}0 & \text { if } \int_{\Omega} \int_{0}^{T} \mathbf{d}: \boldsymbol{\sigma}^{E} d v d t=1 \\ \infty & \text { otherwise }\end{cases}
$$

We also have

$$
\sup _{\widetilde{\boldsymbol{\sigma}} \in \mathcal{C}} \int_{\Omega} \int_{0}^{T} \mathbf{d}(\mathbf{x}, t): \widetilde{\boldsymbol{\sigma}}(\mathbf{x}, t) d v d t=\int_{\Omega} \int_{0}^{T} \mathcal{D}(\mathbf{d}(\mathbf{x}, t)) d v d t
$$

where $\mathcal{D}(\mathbf{d})=\sup _{\boldsymbol{\sigma} \in \mathcal{C}} \boldsymbol{\sigma}: \mathbf{d}$. Let us focus on the last term in (4.4). We denote by $\mathcal{B}_{0}$ the set of strain fields that derive from displacement fields vanishing on $\Gamma_{u}$, i.e.

$$
\mathcal{B}_{0}=\left\{\varepsilon: \varepsilon=\frac{1}{2}\left(\nabla \xi^{r}+\nabla^{t} \xi^{r}\right) ; \xi^{r}=0 \text { on } \Gamma_{u}\right\}
$$

Let $\boldsymbol{\rho}^{r}$ and $\varepsilon^{r}$ be the residual stress and strain fields associated with the strain field $\mathbf{E}^{p}$, as defined by the relations $\rho^{r} \in \mathcal{A}_{0}, \varepsilon^{r} \in \mathcal{B}_{0}$ and

$$
\varepsilon^{r}=\mathbb{L}^{-1}: \rho^{r}+\mathbf{E}^{p}
$$

Hence, for any $\boldsymbol{\rho}^{*} \in \mathcal{A}_{0}$

$$
0=\int_{\Omega} \boldsymbol{\rho}^{*}: \mathbb{L}^{-1}: \boldsymbol{\rho}^{r} d v+\int_{\Omega} \boldsymbol{\rho}^{*}: \mathbf{E}^{p} d v
$$

Choosing $\boldsymbol{\rho}^{*}=x \boldsymbol{\rho}^{r}$ for any arbitrary $x$ shows that

$$
\sup _{\boldsymbol{\rho}^{*} \in \mathcal{A}_{0}}-\int_{\Omega} \mathbf{E}^{p}(\mathbf{x}): \boldsymbol{\rho}^{*}(\mathbf{x}) d v=\infty \quad \text { if } \boldsymbol{\rho}^{r} \neq 0
$$

On the other hand, it follows directly from (4.5) that

$$
\sup _{\boldsymbol{\rho}^{*} \in \mathcal{A}_{0}}-\int_{\Omega} \mathbf{E}^{p}(\mathbf{x}): \boldsymbol{\rho}^{*}(\mathbf{x}) d v=0 \quad \text { if } \boldsymbol{\rho}^{r}=0
$$

The condition $\boldsymbol{\rho}^{r}=0$ means that $\mathbf{E}^{p} \in \mathcal{B}_{0}$. Collecting the results established so far, we have

$$
\sup _{\left(m, \boldsymbol{\rho}^{*}, \widetilde{\boldsymbol{\sigma}}\right) \in \mathcal{A}} \mathcal{L}= \begin{cases}\int_{\Omega} \int_{0}^{T} \mathcal{D}(\mathbf{d}(\mathbf{x}, t)) d v d t & \text { if } \int_{0}^{T} \int_{\Omega} \mathbf{d}: \boldsymbol{\sigma}^{E} d v d t=1 \text { and } \int_{0}^{T} \mathbf{d} \in \mathcal{B}_{0} \\ +\infty & \text { otherwise }\end{cases}
$$

Hence

$$
m_{K}=\inf \left\{\int_{\Omega} \int_{0}^{T} \mathcal{D}(\mathbf{d}(\mathbf{x}, t)) d v d t: \int_{0}^{T} \int_{\Omega} \mathbf{d}: \boldsymbol{\sigma}^{E} d v d t=1 \text { and } \int_{0}^{T} \mathbf{d} \in \mathcal{B}_{0}\right\}
$$

If $m_{K}<1$ then by (4.3) and (3.2) we can conclude that shakedown does not occur. The necessary condition for shakedown is thus that $m_{K} \geqslant 1$. We can thus formulate the following 
Shakedown condition 3 (necessary) If shakedown occurs, then $1 \leqslant \int_{\Omega} \int_{0}^{T} \mathcal{D}(\mathbf{d}(\mathbf{x}, t)) d v d t$ for any history $\mathbf{d}$ such that $\int_{0}^{T} \int_{\Omega} \mathbf{d}: \boldsymbol{\sigma}^{E} d v d t=1$ and $\int_{0}^{T} \mathbf{d} \in \mathcal{B}_{0}$.

In practice, an upper bound $m_{K}^{+}$on $m_{K}$ (and therefore on $m_{S}$ ) can be obtained by constructing specific histories $\mathbf{d}$ satisfying the requirements $\int_{0}^{T} \int_{\Omega} \mathbf{d}: \boldsymbol{\sigma}^{E} d v d t=1$ and $\int_{0}^{T} \mathbf{d} \in \mathcal{B}_{0}$.

\section{Application}

As an illustration, the shakedown theorems presented previously are applied to a problem related to Li-ion batteries. We consider a spherical electrode particle with radius $R$. A chemical potential $^{1} M_{d}(t)$ and pressure $P(t)$ are applied on the boundary as

$$
P(t)=p \cos (\omega t) \quad M_{d}=\mu_{0}+\mu_{d} \cos (\omega t+\phi)
$$

The objective is to find the set of values $\left(p, \mu_{d}, \omega, \phi\right)$ for which elastic shakedown occurs.

The free energy $w$ is taken as isotropic, so that constitutive relations (2.6) become

$$
\sigma_{m}=K \operatorname{tr} \varepsilon+a c \quad \mathbf{s}=2 G\left(\varepsilon^{d}-\varepsilon^{p}\right) \quad \mu=\mu_{0}+k c+a \operatorname{tr} \varepsilon
$$

where $\varepsilon^{d}$ is the deviatoric strain, $\sigma_{m}=(\operatorname{tr} \sigma) / 3$ is the hydrostatic stress, $\mathbf{s}$ is the deviatoric stress and $c$ is the concentration of lithium ions. In (5.2), K, G, $a$ and $\mu_{0}$ are material parameters. The scalar material parameter $a$ in (5.2) accounts for the chemo-mechanical coupling and is directly related to the volumetric expansion coefficient associated with the insertion of lithium. Fick's law is adopted for diffusion, i.e. $\jmath=(D / k) \nabla \mu$ where $D$ is the diffusion coefficient. The elasticity domain $\mathcal{C}$ is of the von Mises type, i.e. defined by $\mathbf{s}: \mathbf{s} \leqslant \sigma_{Y}^{2}$ where $\sigma_{Y}$ is the yield strength.

\subsection{Purely elasto-diffusive response}

The first step consists in finding a $T$-periodic solution $\left(\boldsymbol{\sigma}^{E}, \mu^{E}, \mathbf{u}^{E}, c^{E}\right)$ to the pure elastodiffusive problem. For harmonic loading (5.1), such a $T$-periodic solution $\left(\boldsymbol{\sigma}^{E}, \mu^{E}, \mathbf{u}^{E}, c^{E}\right)$ can be found in the form

$$
\begin{array}{ll}
\boldsymbol{\sigma}^{E}(\mathbf{x}, t)=\Re\left(\widehat{\boldsymbol{\sigma}}^{E}(\mathbf{x}) \mathrm{e}^{\mathrm{i} \omega t}\right) & \mu^{E}(\mathbf{x}, t)=\mu_{0}+\Re\left(\widehat{\mu}^{E}(r) \mathrm{e}^{\mathrm{i} \omega t}\right) \\
J^{E}(\mathbf{x}, t)=\Re\left(\widehat{\jmath}^{E}(\mathbf{x}) \mathrm{e}^{\mathrm{i} \omega t}\right) & \mathbf{u}^{E}(\mathbf{x}, t)=\Re\left(\widehat{u}^{E}(r) \mathrm{e}^{\mathrm{i} \omega t} \mathbf{e}_{r}\right) \\
c^{E}(\mathbf{x}, t)=\Re\left(\widehat{c}^{E}(r) \mathrm{e}^{\mathrm{i} \omega t}\right) &
\end{array}
$$

Here and in the following, the superscript $(\cdot)$ is used to denote complex-values quantities. The real part of complex-valued quantities is denoted by $\Re$. In (5.3), $\left(\mathbf{e}_{r}, \mathbf{e}_{\theta}, \mathbf{e}_{\phi}\right)$ is the local basis for the spherical coordinates $(r, \theta, \phi)$. Substituting (5.3) into the conservation equations and in the constitutive equations leads to a spherical Bessel differential equation in $\widehat{c}^{E}$

$$
r^{2} \frac{d^{2} \widehat{c}^{E}}{d r^{2}}+2 r \frac{d \widehat{c}^{E}}{d r}+\widehat{\lambda}^{2} r^{2} \widehat{c}^{E}=0
$$

where

$$
\widehat{\lambda}=\mathrm{e} 3 \pi / 4 \sqrt{\frac{\omega}{D(1-a \widetilde{a} / k)}} \quad \widetilde{a}=\frac{3 a}{3 K+4 G}
$$

Bounded solutions to (5.4) take the form $\widehat{c}^{E}(r)=A j_{0}(\lambda r)$ where $j$ is the spherical Bessel function of the first kind. The multiplicative constant $A$ as well as the expression of the displacement are

\footnotetext{
${ }^{1}$ For electrode materials, the chemical potential is proportional to the electric voltage. The chemical boundary condition considered thus amounts to prescribe a time-harmonic voltage on the boundary.
} 
obtained from the equilibrium equation and the boundary conditions. Omitting the detail of the calculation, we obtain

$$
A=\frac{\mu_{d} \mathrm{e}^{\mathrm{i} \phi}+\frac{a p}{K}}{(k-a \widetilde{a}) j_{0}(\widehat{R})-\frac{4 G}{K} a \widetilde{a} \frac{j_{1}(\widehat{R})}{\widehat{R}}}
$$

For later reference, we report the final expression of the deviatoric part $\mathbf{s}^{E}$ of the stress response $\boldsymbol{\sigma}^{E}$. Setting $\widehat{r}=\widehat{\lambda} r$ and $\widehat{R}=\widehat{\lambda} R$, we find

$$
\mathbf{s}^{E}(\mathbf{x}, t)=\mathbf{s}_{\theta}^{E}(r, t)\left(-2 \mathbf{e}_{r} \otimes \mathbf{e}_{r}+\mathbf{e}_{\theta} \otimes \mathbf{e}_{\theta}+\mathbf{e}_{\phi} \otimes \mathbf{e}_{\phi}\right)
$$

where $\mathbf{s}_{\theta}^{E}(\mathbf{x}, t)=\Re\left(\widehat{s}_{\theta}^{E}(r) \mathrm{e}^{\mathrm{i} \omega t}\right)$ and

$$
\widehat{s}_{\theta}^{E}(r)=-2 G A \widetilde{a}\left(\frac{j_{1}(\widehat{r})}{\widehat{r}}-\frac{j_{0}(\widehat{r})}{3}\right)
$$

\subsection{Application of the static theorem}

A lower bound on $m_{S}$ can be obtained by taking $\boldsymbol{\rho}=\mathbf{0}$ in (3.1). We have indeed $m_{S} \geqslant m$ for any $m$ such that $0.5 m^{2} \mathbf{s}^{E}(\mathbf{x}, t): \mathbf{s}^{E}(\mathbf{x}, t) \leqslant \sigma_{Y}^{2}$ for all $\mathbf{x}$ and $t$. It follows that

$$
m_{S} \geqslant \frac{\sqrt{2} \sigma_{Y}}{\sqrt{\sup _{\mathbf{x}, t} \mathbf{s}^{E}(\mathbf{x}, t): \mathbf{s}^{E}(\mathbf{x}, t)}}
$$

Direct calculation gives $\sqrt{\mathbf{s}^{E}(\mathbf{x}, t): \mathbf{s}^{E}(\mathbf{x}, t)}=\sqrt{6}\left|\mathbf{s}_{\theta}^{E}(r, t)\right|$. Since $\mathbf{s}_{\theta}^{E}(\mathbf{x}, t)=\Re\left(\widehat{s}_{\theta}^{E}(r) \mathrm{e}^{\mathrm{i} \omega t}\right)$, we have $\sup _{t}\left|\mathbf{s}_{\theta}^{E}(r, t)\right|=\left|\widehat{s}_{\theta}^{E}(r)\right|$. Furthermore, it can be verified that $\left|\widehat{s}_{\theta}^{E}(r)\right|$ increases with $r$. Hence

$$
m_{S} \geqslant \frac{\sigma_{Y}}{\sqrt{3}\left|\widehat{s}_{\theta}^{E}(R)\right|}
$$

\subsection{Application of the kinematic theorem}

Recalling that $\mathbf{s}_{\theta}^{E}(r, t)=\Re\left(\widehat{s}_{\theta}^{E}(r) \mathrm{e}^{\mathrm{i} \omega t}\right)$, we denote by $t_{0}$ and $t_{1}$ the time instants in $[0, T]$ such that $\mathbf{s}_{\theta}^{E}\left(R, t_{0}\right)=-\mathbf{s}_{\theta}^{E}\left(R, t_{1}\right)=\left|\widehat{s}_{\theta}^{E}(R)\right|$. We denote by $\delta_{x}$ the Dirac distribution centered at $x$. Let us consider histories $\mathbf{d}_{n}$ of the form

$$
\mathbf{d}_{n}(\mathbf{x}, t)=f_{n}(r) g_{n}(t)\left(-2 \mathbf{e}_{r} \otimes \mathbf{e}_{r}+\mathbf{e}_{\theta} \otimes \mathbf{e}_{\theta}+\mathbf{e}_{\phi} \otimes \mathbf{e}_{\phi}\right)
$$

where

$$
\begin{array}{lll}
f_{n} \geqslant 0 & \int_{0}^{R} 4 \pi r^{2} f_{n}(r) d r=1 & 4 \pi r^{2} f_{n} \underset{n \rightarrow \infty}{\longrightarrow} \delta_{R} \\
\int_{0}^{T} g_{n}(t) d t=0 & \int_{0}^{T}\left|g_{n}(t)\right| d t=1 & g_{n} \underset{n \rightarrow \infty}{\longrightarrow} \frac{1}{2}\left(\delta_{t_{0}}-\delta_{t_{1}}\right)
\end{array}
$$

Observe that $\int_{0}^{T} \mathbf{d}(\mathbf{x}, t) d t=0$ hence $\int_{0}^{T} \mathbf{d}(\mathbf{x}, t) d t \in \mathcal{B}_{0}$. For the von Mises plasticity model, we have $\mathcal{D}(\mathbf{d})=\sqrt{2} \sigma_{Y}\|\mathbf{d}\|$ if $\mathbf{d}$ is deviatoric, $\mathcal{D}(\mathbf{d})=\infty$ otherwise. Noting that $\operatorname{tr} \mathbf{d}_{n}=0$ we find

$$
\int_{\Omega} \int_{0}^{T} \mathcal{D}\left(\mathbf{d}_{n}(\mathbf{x}, t)\right) d v d t=2 \sqrt{3} \sigma_{Y}\left(\int_{0}^{R}\left|f_{n}(r)\right| 4 \pi r^{2} d r\right)\left(\int_{0}^{T}\left|g_{n}(t)\right| d t\right)=2 \sqrt{3} \sigma_{Y}
$$


Set $I_{n}=\int_{\Omega} \int_{0}^{T} \boldsymbol{\sigma}^{E}: \mathbf{d}_{n} d v d t$. Using the strain history $\mathbf{d}=\mathbf{d}_{n} / I_{n}$ in (4.6) yields

$$
m_{K} \leqslant \frac{2 \sqrt{3} \sigma_{Y}}{I_{n}}
$$

From (5.9) we have

$$
I_{n} \underset{n \rightarrow \infty}{\longrightarrow} 3\left(\mathbf{s}_{\theta}^{E}\left(R, t_{0}\right)-\mathbf{s}_{\theta}^{E}\left(R, t_{1}\right)\right)=6\left|\widehat{s}_{\theta}^{E}(R)\right|
$$

Hence

$$
m_{K} \leqslant \frac{\sqrt{3} \sigma_{Y}}{3\left|\widehat{s}_{\theta}^{E}(R)\right|}
$$

Comparing (5.8) with (5.10) and recalling that $m_{S} \leqslant m_{K}$, we can conclude that $m_{S}=m_{K}=$ $\sigma_{Y} /\left(\sqrt{3}\left|\hat{s}_{\theta}^{E}(R)\right|\right)$. The combination of the static and kinematic theorems thus allows the exact value of $m_{S}$ to be found. From (3.2), the shakedown domain of the space of loading parameters $\left(p, \mu_{d}, \omega, \phi\right)$ is characterized by the equation

$$
\frac{\sigma_{Y}}{\sqrt{3}\left|\widehat{s}_{\theta}^{E}(R)\right|}>1
$$

Consider the dimensionless loading parameters $\left(\widetilde{\mu}_{d}, \widetilde{p}\right)$ defined as $\widetilde{\mu}_{d}=\mu_{d} / a$ and $\widetilde{p}=p / K$. Using (5.7), condition (5.11) can be rewritten in a more explicit fashion as

$$
\widetilde{\mu}_{d}^{2}+\widetilde{p}^{2}+2 \cos \phi \widetilde{\mu}_{d} \widetilde{p} \leqslant H(\omega)^{2}
$$

where

$$
H(\omega)=\frac{\sqrt{3} \sigma_{Y}}{2 G}\left|\frac{\frac{k}{a \widetilde{a}}-1-\frac{4 G}{3 K} z}{1-z}\right| \quad z=\frac{3 j_{1}(\widehat{R})}{\widehat{R} j_{0}(\widehat{R})}
$$

(a)

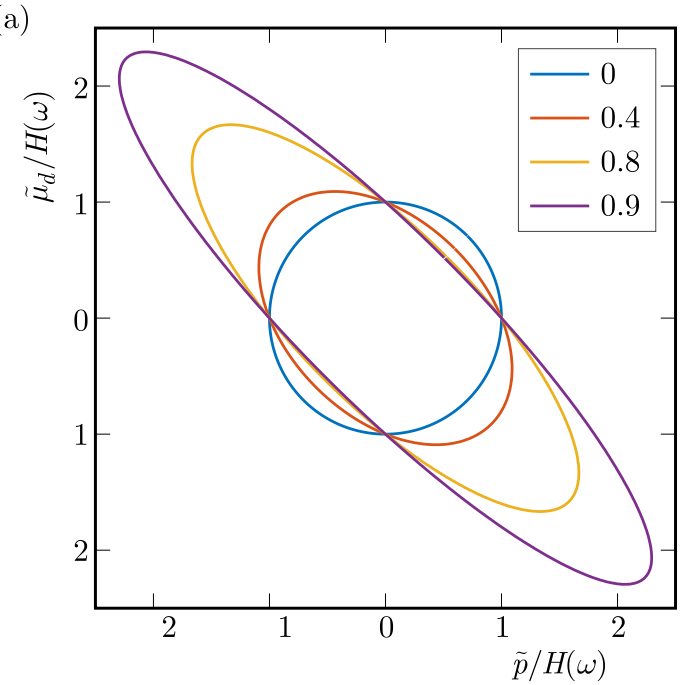

(b)

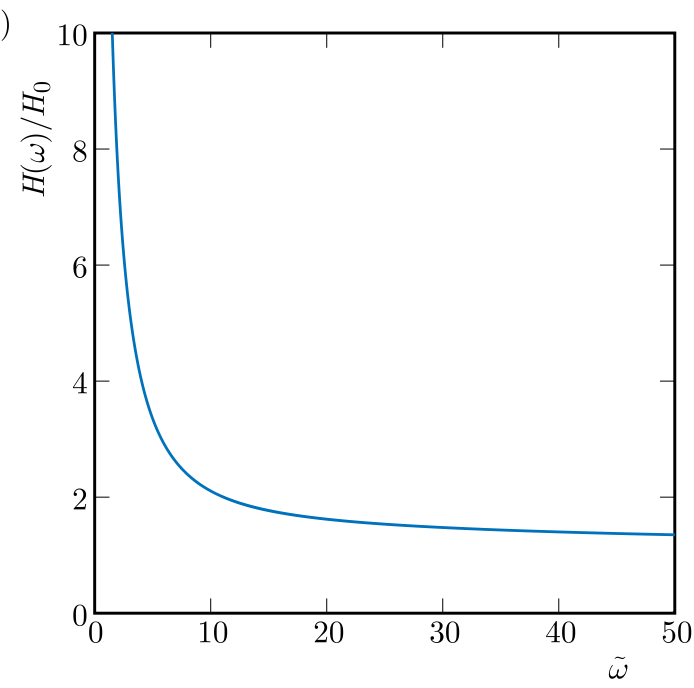

Fig. 1. Shakedown domain $\mathcal{E}(\omega, \phi)$ in the plane $\left(\widetilde{p}, \widetilde{\mu}_{d}\right)$, for $\cos \phi \in\{0,0.4,0.8,0.9\}$ (a). Function $H(\omega)$ defining the size of $\mathcal{E}(\omega, \phi)$ (b)

Equation (5.12) defines the set of loading parameters $\left(\widetilde{p}, \widetilde{\mu}_{d}, \omega, \phi\right)$ for which elastic shakedown occurs. Let $\mathcal{E}(\omega, \phi)$ be the values of $\left(\widetilde{p}, \widetilde{\mu}_{d}\right)$ satisfying (5.12) for any given $(\omega, \phi)$. Provided $|\cos \phi| \neq 1, \mathcal{E}(\omega, \phi)$ has an elliptic shape in the plane $\left(\widetilde{p}, \widetilde{\mu}_{d}\right)$ as represented in Fig. 1a. The size 
of the ellipse $\mathcal{E}(\omega, \phi)$ is controlled by the function $H(\omega)$. That function is represented in Fig. 1b in terms of the dimensionless charging rate $\widetilde{\omega}=\omega R^{2} / D$. The plot in Fig. 1b corresponds to the situation $a \widetilde{a} \ll k$ in which case $H(\omega) \simeq H_{0} /|z-1|$ with $H_{0}=\sqrt{3} \sigma_{Y} k /(2 G a \widetilde{a})$.

Two main observations are in order. The first one is that the applied pressure is found to have an influence on the shakedown behavior. This is in contrast with diffusion-less plasticity, for which the stress in a spherical particle under pressure is purely hydrostatic. The second observation is that, even though the rate-independent plasticity is considered, the loading rate has an influence on the shakedown limit (through the function $H$ ). This is a consequence of the coupling between plasticity and diffusion, the latter being a rate-dependent process. Note that $H(\omega)$ becomes infinite in the limit $\omega \rightarrow 0$. This corresponds to situations in the lithium ions have time to diffuse uniformly in the whole particle.

\section{References}

1. Brassart L., Zhao K., Suo Z., 2013, Cyclic plasticity and shakedown in high-capacity electrodes of lithium-ion batteries, International Journal of Solids and Structures, 50, 1120-1129

2. Klarbring A., Barber J.R., Spagnoli A., Terzano M., 2017, Shakedown of discrete systems involving plasticity and friction, European Journal of Mechanics - A/Solids, 64, 160-164

3. Koiter W.T., 1960, General theorems for elastic-plastic solids, [In:] Progress in Solid Mechanics, I.N. Sneddon, R. Hill (Edit.), 4, 165-221

4. LARChÉ F., CAhN J.W., 1973, A linear theory of thermochemical equilibrium of solids under stress, Acta Metallurgica, 21, 1051-1063

5. Melan E., 1936, Theorie statisch unbestimmter Systeme aus ideal-plastischen Baustoff, Sitz. Berl. Ak. Wiss., 145, 195-218

6. NGUYen Q.S., 2003, On shakedown analysis in hardening plasticity, Journal of the Mechanics and Physics of Solids, 51, 101-125

7. Peigney M., 2010, Shakedown theorems and asymptotic behaviour of solids in non-smooth mechanics, European Journal of Mechanics - A/Solids, 29, 784-793

8. Peigney M., 2014a, On shakedown of shape memory alloys structures, Annals of Solid and Structural Mechanics, 6, 17-28

9. Peigney M., 2014b, Shakedown of elastic-perfectly plastic materials with temperature-dependent elastic moduli, Journal of the Mechanics and Physics of Solids, 71, 112-131

10. Peigney M., 2018, Cyclic steady states in diffusion-induced plasticity with applications to lithiumion batteries, Journal of the Mechanics and Physics of Solids, 111, 530-556

11. Pham D.C., 2017, Consistent limited kinematic hardening plasticity theory and path-independent shakedown theorems, International Journal of Mechanical Sciences, 130, 11-18 